\title{
1 An IH-QCL based gas sensor for simultaneous detection of methane and acetylene
}

2

4

$5 \quad *$ Corresponding author email: aamir.farooq@kaust.edu.sa

6

7

8 Colloquium: Diagnostics

9

10

11 Word count (using Method 1):

12 Total: 6102 words

13 Main text: 3387

14 Reference: $542($ Word count $=(27+2) \times(2.3) \times(7.6)=507)$

15 Equations: 182

16

17 Figure and captions: 2026

18

19 This paper contains Supplementary Material.

\author{
Guangle Zhang, Kuanysh Khabibullin, Aamir Farooq*
}




\section{An IH-QCL based gas sensor for simultaneous detection of methane and acetylene}

22

23

24

25

26

27

28

29

30

31

32

33

34

35

36

37

38

39

40

41

42

Guangle Zhang, Kuanysh Khabibullin, Aamir Farooq*

King Abdullah University of Science and Technology, Clean Combustion Research Center, Physical Sciences and Engineering Division, Thuwal 23955, Saudi Arabia

\section{Abstract}

Extended wavelength tuning of an IH-QCL (integrated heater quantum cascade laser) is exploited for simultaneous detection of methane and acetylene using direct absorption spectroscopy. The integrated heater, placed within few microns of the laser active region, enables wider wavelength tuning than would be possible with a conventional DFB (distributed feedback) QCL. In this work, the laser current and heater resistor current are modulated simultaneously at $25 \mathrm{kHz}$ to tune the laser over $1279.6-1280.1 \mathrm{~cm}^{-1}$, covering absorption transitions of methane and acetylene. The laser is characterized extensively to understand the dependence of wavelength tuning on modulation frequency, modulation amplitude and phase difference between laser / heater modulation. Thereafter, the designed sensor is validated in roomtemperature static cell experiments. Finally, the sensor is applied for simultaneous detection of methane and acetylene during the high-temperature pyrolysis of iso-octane behind reflected shock waves.

Keywords: QCL; Shock tube; Methane; Acetylene; Absorption spectroscopy 


\section{Introduction}

44 As a virtually ideal coherent source in the mid-infrared wavelength range, quantum cascade laser (QCL)

45 has become increasingly popular for gas sensing in a variety of fields such as environmental monitoring

46 [1-3], industrial process control $[4,5]$, biomedical devices [6, 7] and combustion diagnostics [8, 9]. A

47 QCL emits radiation by using inter-subband transitions through a stack of quantum wells [10], which are

48 realized by burying quantum heterostructures into the laser structure. To obtain stable single mode

49 emission, a distributed feedback (DFB) grating is usually integrated in the buried heterostructure, which

50 forms a DFB QCL. Usually, this DFB QCL has limited electrical tunability, typically $\sim 0.1-0.2 \%$ of the

51 central frequency. Although the tuning range can be a few times wider by changing the substrate

52 temperature of the laser, the tuning speed is limited to $\mathrm{Hz}$ range when changing the substrate temperature

53 in contrast to tens of $\mathrm{kHz}$ tuning rate with electrical tuning. To achieve a wide tuning range as well as a

54 high tuning speed, Bismuto et al. [11] placed an integrated heater (IH) very close (within a few microns)

55 to the laser active region (AR). This arrangement allowed the control of AR temperature much faster by

56 changing the current injected into the IH. Bismuto et al. [11] demonstrated wider tuning capability of the

57 IH-QCL concept at wavelengths near $7.8 \mu \mathrm{m}$ and $4.9 \mu \mathrm{m}$, and achieved frequency tuning up to $0.5 \%$ of

58 the central frequency. Such a device presents new opportunities for molecular spectroscopy in the mid-IR

59 region to achieve broader tuning for detecting many species simultaneously or for broadband absorbers.

60 Although, commercial external-cavity QCLs can provide relatively large wavelength access in the mid-

61 IR region, the tuning is achieved by mechanical movement of a grating which limits the tuning speed to

$62 \sim 350 \mathrm{~Hz}$ [12]. Another widely tuning source is a super-continuum laser but such lasers are not yet

63 available in the mid-IR beyond $\sim 4.2 \mu \mathrm{m}[13]$ and measurements carried out with super-continuum sources

64 typically require significant averaging to suppress intensity fluctuations. 
65 In this work, we utilize the new IH-QCL technology to develop a gas sensor for combustion applications.

66 We chose an IH-QCL, emitting near $7.8 \mu \mathrm{m}$, which was readily available at Alpes Lasers and its

67 performance had been verified in reference [11]. The wavelength range accessible by this laser has

68 absorption features of nitrous oxide $\left(\mathrm{N}_{2} \mathrm{O}\right)$, methane $\left(\mathrm{CH}_{4}\right)$ and acetylene $\left(\mathrm{C}_{2} \mathrm{H}_{2}\right)$. Here, we selected

69 methane and acetylene as target molecules, mainly because these are very important intermediate species

70 formed during the pyrolysis and oxidation of hydrocarbon fuels. Simultaneous detection of these two

71 species with high accuracy can help validate the chemical kinetic models and constrain the rate constants

72 of critical reaction steps.

73 Previously, methane sensing based on QCL absorption spectroscopy has been demonstrated in

74 atmospheric and plasma applications $[14,15]$. Acetylene detection in the mid-IR with QCL absorption

75 spectroscopy is quite scarce [16]. Sajid et al. [17, 18] demonstrated highly time-resolved measurements

76 of methane and acetylene at high temperatures by using an external cavity quantum cascade laser (EC-

77 QCL). However, due to the slow wavelength-scanning speed of the EC-QCL, they were unable to measure

$78 \mathrm{CH}_{4}$ and $\mathrm{C}_{2} \mathrm{H}_{2}$ simultaneously; the EC-QCL had to be operated separately at two different wavelengths

79 for $\mathrm{CH}_{4}$ and $\mathrm{C}_{2} \mathrm{H}_{2}$ measurements. Here, we use the IH-QCL to realize simultaneous, time-resolved

80 measurements of $\mathrm{CH}_{4}$ and $\mathrm{C}_{2} \mathrm{H}_{2}$ during the high-temperature pyrolysis of iso-octane in a shock tube. To

81 our knowledge, this is the first application of an IH-QCL architecture for combustion diagnostics.

82

83

84

85

86 


\section{Measurement principles}

88

The sensor presented in this work is based on direct absorption spectroscopy which is described by the Beer-Lambert law. The absorbance $\alpha_{i j}$ of a spectroscopic transition $i$ for a species $j$ is given by,

$$
-\ln \left(\frac{I_{t}}{I_{0}}\right) \equiv \alpha_{i j}\left(v_{i}, \mathrm{~T}, \mathrm{P}\right)=\frac{P \cdot L}{R \cdot T} \cdot X_{j} \cdot \sigma_{i j}\left(v_{i}, \mathrm{~T}, \mathrm{P}\right)
$$

where $I_{t}$ is the transmitted laser intensity, $I_{0}$ the zero-absorption laser intensity, $v_{i}$ the wavenumber $\left(\mathrm{cm}^{-1}\right)$, $T$ the temperature (K), $P$ the total pressure (bar), $L$ the absorption path length $(\mathrm{m}), R$ the gas constant $(=$ $\left.8.3144598 \mathrm{~m}^{3} \cdot \mathrm{bar}^{-1} \mathrm{~K}^{-1} \cdot \mathrm{mol}^{-1}\right), X_{j}$ the mole fraction of species $j, \sigma_{i j}$ the absorption cross-section $\left(\mathrm{m}^{2} / \mathrm{mol}\right)$ at frequency $v_{i}$.

The IH-QCL used in this work is able to access $1276 \mathrm{~cm}^{-1}$ to $1284 \mathrm{~cm}^{-1}$ region, which contains several absorption transitions of methane and acetylene. In order to select suitable detection wavelengths, homogeneous-reactor chemical kinetic simulations were carried out for the pyrolysis of is-octane, a primary reference fuel, by using the detailed kinetic mechanism of Mehl et al. [19] in Chemkin-Pro [20]. A representative simulation result is shown in Fig. S1 (Supplementary Material). It may be observed that the primary products of iso-octane pyrolysis are $\mathrm{CH}_{4}, \mathrm{C}_{2} \mathrm{H}_{2}, \mathrm{H}_{2}, \mathrm{C}_{2} \mathrm{H}_{4}, \mathrm{C}_{3} \mathrm{H}_{6}, \mathrm{C}_{2} \mathrm{H}_{6}$, and $\mathrm{C}_{4} \mathrm{H}_{8}$. Among the product species, $\mathrm{H}_{2}$ is not infrared active. Spectroscopic simulations for other major species were carried out over $1276 \mathrm{~cm}^{-1}$ to $1284 \mathrm{~cm}^{-1}$ using the spectral parameters given in the HITRAN [21] and PNNL [22] spectral database (see Fig. S2). It was found that $\mathrm{C}_{2} \mathrm{H}_{4}, \mathrm{C}_{2} \mathrm{H}_{6}$ and $\mathrm{C}_{3} \mathrm{H}_{6}$ have negligible absorption in the target wavelength region. Iso-butene $\left(\mathrm{C}_{4} \mathrm{H}_{8}\right)$ has broadband spectra in this region; however, the combination of relatively small production of iso-butene (Fig. S1) and its relatively smaller absorption cross-section (Fig. S2) would limit interference absorption by iso-butene. Secondly, the interference by any broadband absorber can easily be mitigated by the 'differential absorption strategy' which is straightforward to implement here due to the scanned-wavelength approach adopted here. Therefore, this wavelength region $\left(1276-1284 \mathrm{~cm}^{-1}\right)$ is quite suitable for the detection of methane and acetylene during 
hydrocarbon pyrolysis. A representative spectroscopic simulation is plotted in Fig. 1, which shows that the target species $\left(\mathrm{CH}_{4}\right.$ and $\left.\mathrm{C}_{2} \mathrm{H}_{2}\right)$ have resolved spectra in this wavelength window. Three simple criteria

112 were used for selecting detection wavelengths: i) maximum peak absorption, ii) minimum interference 113 absorption, iii) minimum wavelength separation between the two species. This process resulted in 114 selecting two detection wavelengths, $1279.67 \mathrm{~cm}^{-1}$ and $1280.01 \mathrm{~cm}^{-1}$, for simultaneous sensing of $\mathrm{CH}_{4}$ 115 and $\mathrm{C}_{2} \mathrm{H}_{2}$, respectively. Figure 1 shows that the $1279.67 \mathrm{~cm}^{-1}$ (labelled $p_{1}$ ) wavelength has some 116 interference absorption by $\mathrm{C}_{2} \mathrm{H}_{2}$. On the other hand, the acetylene detection wavelength, $1280.01 \mathrm{~cm}^{-1}$ 117 (labelled $p_{2}$ ), has negligible interference from methane. Negligible interference by methane at 1280.01 $118 \mathrm{~cm}^{-1}$ was verified by carrying out high-temperature shock tube experiments (not reported here) with $3 \%$ $119 \mathrm{CH}_{4} /$ Ar mixture, and measuring absorption at $p_{2}$.

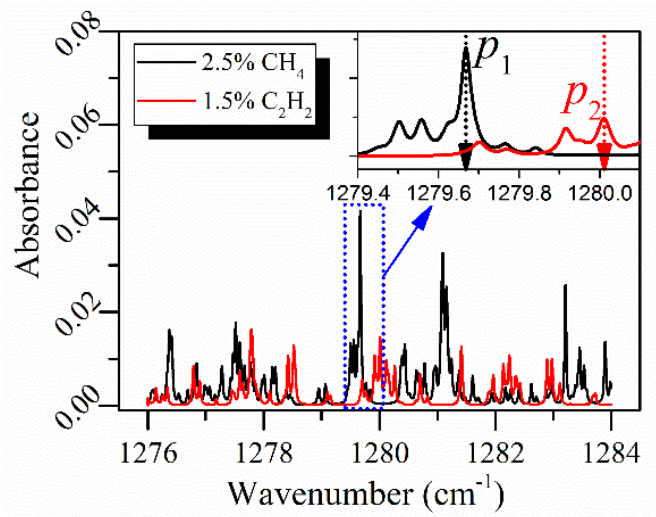

Absorbance at the two selected peaks may be described as:

$$
\left\{\begin{array}{l}
\alpha_{p_{1}}=\frac{P \cdot L}{R \cdot T} \cdot\left(X_{C_{H_{4}}} \cdot \sigma_{C_{4}}^{p_{1}}+X_{C_{2} H_{2}} \cdot \sigma_{C_{2} H_{2}}^{p_{1}}\right) \\
\alpha_{p_{2}}=\frac{P \cdot L}{R \cdot T} \cdot X_{C_{2} H_{2}} \cdot \sigma_{C_{2} H_{2}}^{p_{2}}
\end{array}\right.
$$

124 where $X_{C H 4}, X_{C H 4}$ are the unknowns, i.e., the mole fractions of methane and acetylene, respectively; $\sigma_{C_{2} H_{2}}^{p_{1}}$, $125 \sigma_{C_{2} \mathrm{H}_{2}}^{p_{2}}$ are the cross-section of $\mathrm{C}_{2} \mathrm{H}_{2}$ at the two peaks, and $\sigma_{C H_{4}}^{p_{1}}$ is the cross-section of $\mathrm{CH}_{4}$ at $p_{1}$. The mole 
126 fractions of $\mathrm{CH}_{4}$ and $\mathrm{C}_{2} \mathrm{H}_{2}$ can be determined by solving Eq. (2). It should be noted that the absorption 127 cross-sections are generally a function of temperature, pressure and mixture composition.

\section{Experimental details}

The IH-QCL (integrated heater - quantum cascade laser) used here was provided by Alpes Lasers in a high-heat load (HHL) packaging. The substrate temperature of the laser was controlled by a thermoelectric cooler (TEC) over -15 to $50 \mathrm{C}$. Some operating conditions required the HHL to be kept below room temperature; therefore, the laser was mounted on a copper plate which was cooled by a recirculating chiller

(ThermoCube 400). The current injected into the laser was controlled by two current controllers (ILX Lightwave LDX-3232); one for the AR (active region) current, and the other for IH (integrated heater) current. The maximum allowable AR and IH currents were $225 \mathrm{~mA}$ and $600 \mathrm{~mA}$, respectively. The maximum output power of the laser was $\sim 80 \mathrm{~mW}$. A function generator (LeCroy 2052) was used to drive the two current controllers with waveforms of desired shape, frequency, amplitude and phase difference (between the two waveforms).

Optical setup used for shock tube experiments is shown in Fig. 2. The shock tube facility has been described in detail previously [23-25]. The laser was divided in two beams by a beam splitter (Thorlabs BSW710). One beam was aligned to a wavelength-meter (Bristol Instruments 621-XIR) for absolute frequency measurement or to a Germanium etalon $\left(\mathrm{FSR}=0.0164 \mathrm{~cm}^{-1}\right)$ for monitoring the wavelength tuning. The second beam was propagated through the shock tube via ZnSe optical windows, installed at 2 $\mathrm{cm}$ from the shock tube end-wall. A plano-convex lens $\left(\mathrm{CaF}_{2}\right.$, focal length of $\left.200 \mathrm{~mm}\right)$ and an iris, upstream of the shock tube, were used to minimize beam steering effects caused by density gradients

147 during high-temperature experiments. A second iris, downstream of the shock tube, was used to reduce 148 the undesired broadband thermal emission caused by hot gases. A concave mirror (focal length of $5 \mathrm{~mm}$ ) 
was utilized to focus the laser beam onto a photo-detector (Vigo Systems PVI-2TE-10.6 2mm $\times 2 \mathrm{~mm}$ ). A signals at a sampling rate of $62.5 \mathrm{MHz}$ with a 15-bit resolution.

152

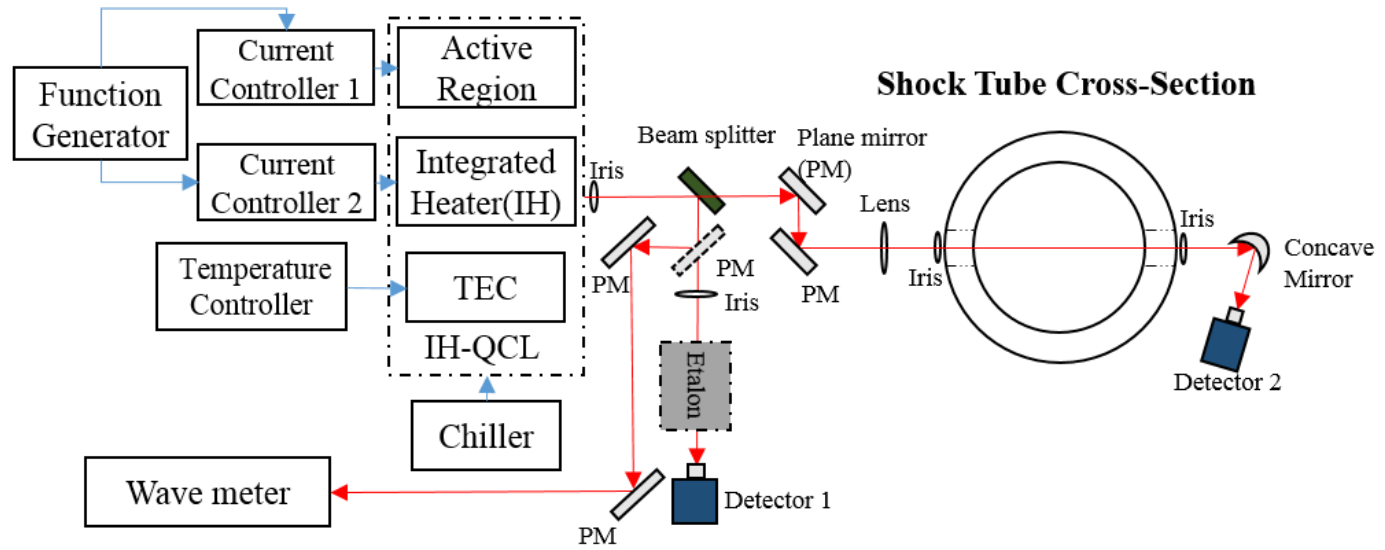

Fig. 2. Optical schematic of the experimental setup.

\section{Laser characterization}

In order to make simultaneous measurements of methane and acetylene at the selected peaks, the IH-QCL is to be scanned $\sim 0.5 \mathrm{~cm}^{-1}$ at a high time resolution (few tens of $\mathrm{kHz}$ ), which meets the requirement of kinetic measurements in a shock tube. Therefore, the laser was characterized to understand how the tuning range is affected by relevant parameters, such as $\mathrm{AR} / \mathrm{IH}$ tuning, modulation frequency of the injected currents, type of modulation wave, and phase difference between the respective modulation waves for IH (integrated heater) tuning and AR (active region) tuning. The first two factors were examined by operating the laser at various modulation frequencies in three different tuning manners, AR tuning, IH tuning and simultaneous $\mathrm{AR} / \mathrm{IH}$ tuning. The tuning range was measured by using the Ge etalon, as described in

Section 3. Figure 3 shows that the tuning range is larger when both AR and IH current are modulated simultaneously, although the incremental tuning achieved by $\mathrm{AR}+\mathrm{IH}$ modulation decreases at faster modulation speeds. The tuning range was characterized at different substrate temperatures and with 
various waveform shapes, such as ramp signal with varying duty cycles, triangular wave, and sinusoidal wave. Among these, a sinusoidal wave provided maximum tuning range, followed by a triangular waveform.

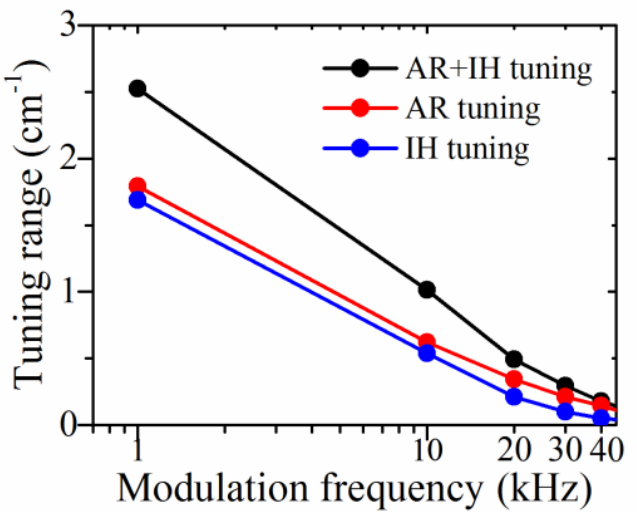

Fig. 3. Wavelength tuning range of the IH-QCL at different modulation frequencies in three different tuning manners. The substrate temperature of the laser was set at $5{ }^{\circ} \mathrm{C}$, and the chiller was set at $15^{\circ} \mathrm{C}$. Ramp signal (90:10) was used as the modulation wave. For simultaneous AR and IH tuning, the phase difference between the two waveforms was $0^{\circ}$.

Since simultaneous AR and IH tuning leads to increased wavelength tuning, the differences in phase and the frequency of two modulation waves were investigated to see their effects on laser performance. It was found that when the two waves were not modulated at the same frequency, the laser intensity was not stable. Yet, a stable laser intensity signal is necessary for direct absorption experiments, therefore, the same modulation frequency was used for the two waves. For the phase difference investigation, the phase in IH current wave was fixed at $0^{\circ}$ while the phase in AR current wave was changed from $0^{\circ}$ to $360^{\circ}$. The results are shown in Fig. 4. It may be observed that maximum tuning range was achieved when the two waves were in phase. The tuning range decreased steadily as the phase difference increased, reaching a minimum when the two waves were out of phase. Moreover, as the modulation frequency increased, the minima moved from phase difference of $\sim 180^{\circ}$ to $\sim 130^{\circ}$. This shift may be attributed to the fact that wavelength tuning is thermal in nature, which depends on local values of the thermal resistance and heat expansion as well as chip geometry, and increased modulation frequencies perhaps lead to varying thermal behavior from the two tuning modes (AR and $\mathrm{IH})$. Based on the above laser characterization and required 
tuning range of $\sim 0.5 \mathrm{~cm}^{-1}$, the $\mathrm{AR}$ and $\mathrm{IH}$ currents were both modulated at $25 \mathrm{kHz}$ with a phase difference of zero in the experiments presented in Section 5 and Section 6.

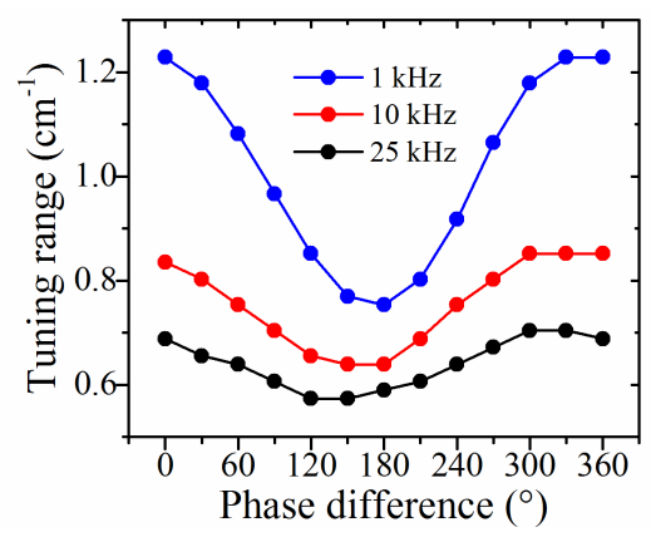

191 192 193 194
Fig. 4. Wavelength tuning range as a function of phase difference between AR and IH tuning waves. Sinusoidal waveforms were used for both tuning modes at $1 \mathrm{kHz}, 10 \mathrm{kHz}$ and $25 \mathrm{kHz}$. The substrate temperature of the laser was set at $5^{\circ} \mathrm{C}$, and the chiller was set at $15^{\circ} \mathrm{C}$. The AR dc current was set to $160 \mathrm{~mA}$ while IH dc current was 300 $m A$.

Additionally, the intensity modulation response of the laser with IH and AR tuning was investigated. The laser was modulated with IH and AR tuning modes separately, and the amplitude of the respective input waveform was adjusted to achieve maximum wavelength tuning response at each modulation frequency. Figure 5 shows that the intensity amplitude decreased steadily for AR modulation as the modulation frequency increased from 1 to $100 \mathrm{kHz}$, while the amplitude remained stable at frequencies higher than $20 \mathrm{kHz}$ for IH modulation. Moreover, the intensity amplitude of IH tuning was much lower than that of AR tuning at the same modulation frequency and approximately same wavelength tuning range. The reduced intensity modulation response can be a very desirable feature for a number of applications. For example, the use of IH modulation for wavelength modulation spectroscopy (WMS) can be quite useful for minimizing the undesired residual amplitude modulation signal [26]. 


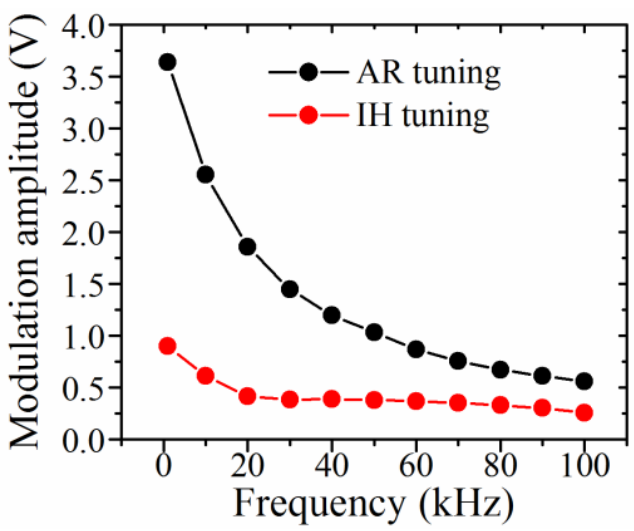

Fig. 5. Modulation amplitude with AR tuning and IH tuning separately. The substrate temperature of the laser was set at $5{ }^{\circ}$ C. Ramp signal (90:10) was used as the modulation wave. Input IH waveform amplitude: 2.9 Vp-p; input AR waveform amplitude: $0.65 \mathrm{Vp}$-p. The chiller was set to $15^{\circ} \mathrm{C}$. The AR dc current was set to $160 \mathrm{~mA}$ while IH dc current was 300 $m A$.

\section{Static cell measurements}

To verify the methodology of simultaneously sensing $\mathrm{CH}_{4}$ and $\mathrm{C}_{2} \mathrm{H}_{2}$, we performed room-temperature (294 K) experiments in a $14 \mathrm{~cm}$ long static cell. The static cell was first vacuumed to get the zeroabsorption signal $\left(I_{0}\right)$. Then it was filled up to various pressures with a mixture of $1.4 \% \mathrm{CH}_{4}, 0.77 \% \mathrm{C}_{2} \mathrm{H}_{2}$ and balance $\mathrm{N}_{2}$. The transmitted laser signal $\left(I_{t}\right)$, with simultaneous $\mathrm{AR}$ and $\mathrm{IH}$ modulation, was then recorded to obtain the absorbance profile. A representative absorbance profile is shown in Fig. S3 (Supplementary Material). Measured mole fractions of methane and acetylene were calculated by using Eq. (2) with the absorption cross-section values taken from the HITRAN database. For a specific experiment carried out at a total pressure of 102 Torr, the laser measurement gave $\mathrm{CH}_{4}$ mole fraction of $1.38 \%$ and $\mathrm{C}_{2} \mathrm{H}_{2}$ mole fraction of $0.79 \%$. These results are in excellent agreement with the manometrically recorded mole fractions. Uncertainty in the measured values comes primarily from uncertainties in the HITRAN spectral parameters, pressure measurement, mixture preparation and minor variations in laser intensity. 


\section{Shock tube measurements}

227 Shock tube experiments are generally performed with argon as the bath gas, since the use of nitrogen bath 228 gas can lead to boundary layer separation and shock bifurcation [27]. The HITRAN database can be used 229 to calculate absorption cross-section values for air (or nitrogen) bath gas but not for argon bath gas. 230 Therefore, for the high-temperature detection of methane and acetylene in the shock tube, the respective absorption cross-sections must be measured experimentally. Methane cross-section measurements were carried out over 1300 - $1750 \mathrm{~K}$ using $3 \% \mathrm{CH}_{4} / \mathrm{Ar}$ mixture, and acetylene cross-sections were measured over $1300-1700 \mathrm{~K}$ using $5 \% \mathrm{C}_{2} \mathrm{H}_{2} / \mathrm{Ar}$ mixture. Fixed-wavelength absorption strategy was employed to achieve high temporal resolution, and the measured absorbance signals were averaged over $1 \mathrm{~ms}$ after the reflected shock wave. A representative laser transmission signal is shown in Fig. S4 (Supplementary

Material), which depicts high signal-to-noise ratio achieved in these experiments. It should be noted that methane and acetylene may start to undergo pyrolysis at high temperatures (>1700 K); absorbance signal at time zero (arrival of reflected shock wave) was used in such cases to determine absorption cross-section.

The measured cross-sections are plotted in Fig. 6, and the best fit values (shown as solid red line) are of cross-sections on pressure for these narrow absorption lines. The uncertainty in cross-section comes 242 from uncertainties of pressure $(<1 \%)$ and temperature $(<1 \%)$ of the reflected shock, path-length in the signal $(\sim 3 \%)$. Gathering all these factors in a root-sum-squared method, uncertainties in measured crosssections range $\sim 3-7 \%$, and are shown as error bars in Fig. 6.

$$
\begin{cases}\sigma_{C_{4}}^{p_{1}}=2 \times 10^{7} \cdot T^{-2.08} \cdot P^{-0.56} ; & (1300-1750 \mathrm{~K}),(0.97-1.3 \mathrm{~atm}) \\ \sigma_{C_{2} H_{2}}^{p_{1}}=59 \cdot T^{-0.44} \cdot P^{-0.077} ; & (1300-1700 \mathrm{~K}),(0.97-1.3 \mathrm{~atm}) \\ \sigma_{C_{2} \mathrm{H}_{2}}^{p_{2}}=3.6 \times 10^{5} \cdot T^{-1.50} \cdot P^{-0.46} ; & (1300-1700 \mathrm{~K}),(0.97-1.3 \mathrm{~atm})\end{cases}
$$



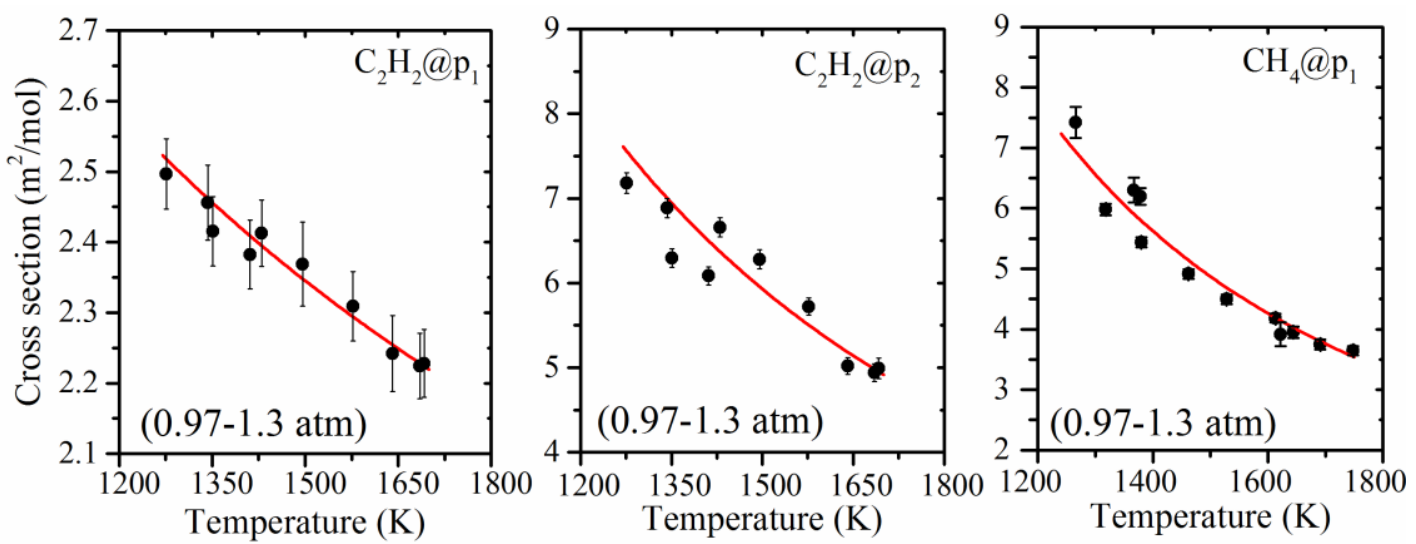

Fig. 6. Measured absorption cross-sections of methane and acetylene at the two selected peaks by using $3 \% \mathrm{CH}_{4} /$ Ar and 5\% $\mathrm{C}_{2} \mathrm{H}_{2} / \mathrm{Ar}$ mixtures. The black circles are the measured data, while the red solid lines represent the fitted results normalized at $1.1 \mathrm{~atm}$. Error bars indicate measurement uncertainty.

Thereafter, shock tube experiments were carried out with mixtures of methane and acetylene to verify the sensing methodology and measured absorption cross-sections. Two different nominal mixtures were used: $3 \% \mathrm{CH}_{4} / 5 \% \mathrm{C}_{2} \mathrm{H}_{2} / \mathrm{Ar}$ and $2 \% \mathrm{CH}_{4} / 3 \% \mathrm{C}_{2} \mathrm{H}_{2} / \mathrm{Ar}$. In these experiments, the laser was modulated at 25 $\mathrm{kHz}$ using simultaneous $\mathrm{AR}$ and $\mathrm{IH}$ tuning to scan over the intended absorption transitions of methane and acetylene. A representative laser transmission signal and its absorbance profile are shown in Fig. S5 (Supplementary Material). The experiments scanned temperature range of $\sim 1300-1700 \mathrm{~K}$ and pressures near 1 atm. Representative measured time-history results are shown in Fig. 7 which have an average absolute deviation of $\sim 6 \%$ compared to the manometrically measured mole fraction values. Since methane undergoes pyrolysis at temperatures $>1600 \mathrm{~K}$, those results are not included here. The uncertainty in $\mathrm{C}_{2} \mathrm{H}_{2}$ mole-fraction comes from the uncertainty of temperature $(<1 \%)$, pressure $(<1 \%)$, path-length $(\sim 1.4 \%)$, the absorbance peak near $p_{2}\left(\alpha_{p_{2}}, \sim 2-4 \%\right)$ and the $\mathrm{C}_{2} \mathrm{H}_{2}$ cross-section near $p_{2}\left(\sigma_{C_{2} H_{2}}^{p_{2}}, \sim 3-5 \%\right)$. Combining the above factors in a root-sum-squared method, the uncertainty in measured $\mathrm{C}_{2} \mathrm{H}_{2}$ mole-fraction is estimated to be $<7 \%$. Three additional factors, i.e., the uncertainty of absorbance peak near $p_{1}\left(\alpha_{p_{1}}, \sim 2-\right.$ $4 \%), \mathrm{C}_{2} \mathrm{H}_{2}$ cross-section near $p_{1}\left(\sigma_{C_{2} \mathrm{H}_{2}}^{p_{1}}, \sim 4-5 \%\right)$ and the $\mathrm{CH}_{4}$ cross-section near $p_{1}\left(\sigma_{C H_{4}}^{p_{1}}, \sim 3-7 \%\right)$, are used to calculate the uncertainty in $\mathrm{CH}_{4}$ mole-fraction, which is estimated to be $<12 \%$. 

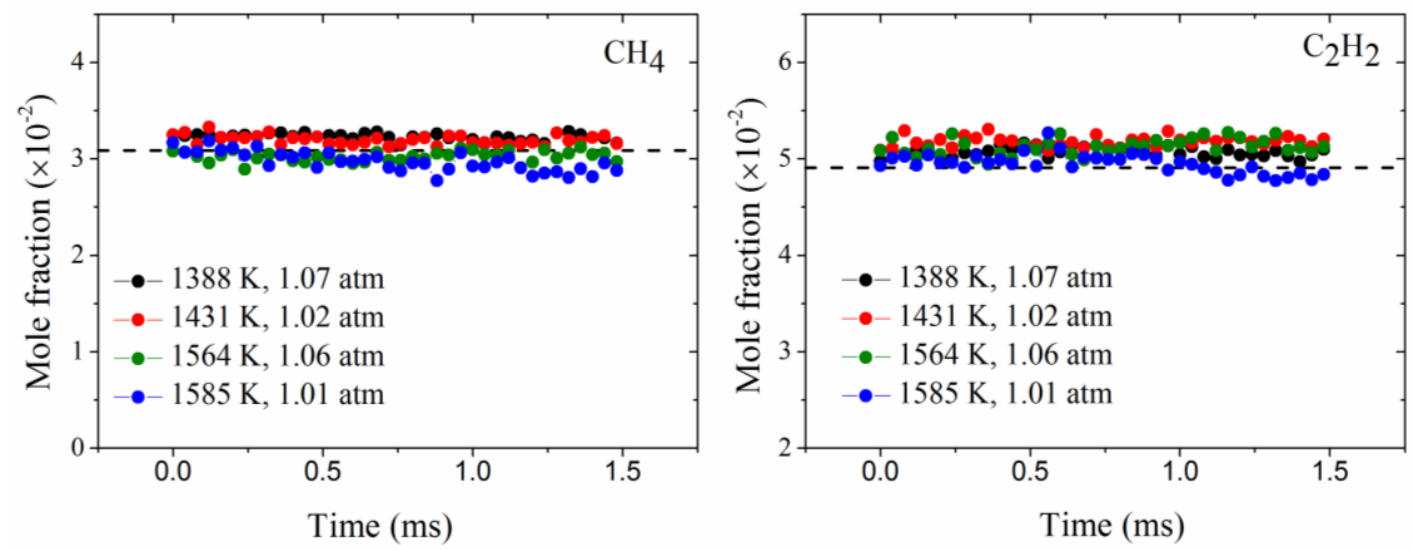

Fig. 7. Measured mole-fraction time-histories for 3.1\% $\mathrm{CH}_{4} / 4.9 \% \mathrm{C}_{2} \mathrm{H}_{2} / \mathrm{Ar}$ mixture. The two black dash lines are the manometric mole fractions while the colorful scatters are the measured results by the sensor.

Finally, the sensor was applied to make simultaneous measurements of methane and acetylene during the pyrolysis of 2\% iso-octane in argon. The pyrolysis process is endothermic and results in a temperature drop after the reflected shock wave. Temperature time-history was obtained from chemical kinetic simulation in Chemkin-Pro [20] by using constant-volume assumption and the detailed kinetic model of Mehl et al. [19]. Previous works [18] have discussed the suitability of using constant-volume or constantpressure constrains in calculating temperature time-histories for shock tube pyrolysis experiments. The simulated temperature time-history was used to calculate time-varying temperature-dependent absorption cross-sections using Eq. (3). Finally, the mole fractions of methane and acetylene were calculated using Eq. (2). A representative absorbance profile is shown in Fig. S6 (Supplementary Material). Two representative results are presented in Fig. 8 and compared with the predictions of Mehl et al. [19] model. To achieve faster time resolution for these kinetic measurements, absorption profiles in both upward and downward scan of sinusoidal wave were used, which resulted in an effective time resolution of $20 \mu$ s. Measured and simulated profiles show that the production of methane and acetylene increases with temperature. The measured mole fractions are generally higher than the predicted ones, which may either be due to deficiencies in the chemical kinetic model for pyrolytic reactions at these conditions or due to some contribution by broadband absorbing species (e.g., iso-butene). The latter effect can be easily 
removed by analyzing the data with differential absorption strategy. For the $1965 \mathrm{~K}$ case in Fig. 8, the measured $\mathrm{CH}_{4}$ mole-fraction exhibits a downward trend between 0.4 and $0.8 \mathrm{~ms}$, which may be caused by some measurement noise, such as beam steering or laser intensity fluctuation. Nevertheless, these results show the potential of this new laser sensor for multi-species measurements in shock tube chemical kinetics experiments.
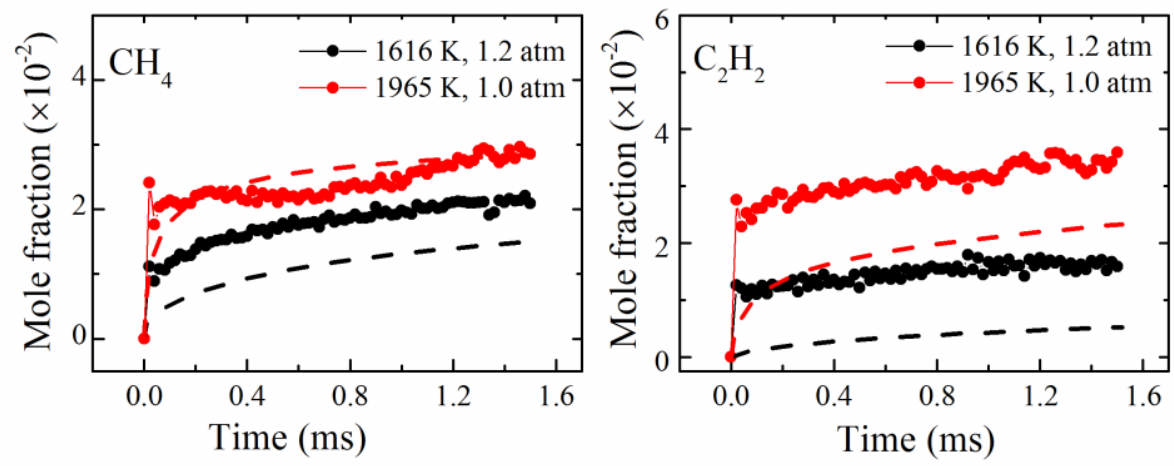

Fig. 8. Mole fraction results of methane and acetylene for two reflected-shock experiments at $1616 \mathrm{~K}, 1.20 \mathrm{bar}$ and 1965 K, 1.01 bar. The dash lines are simulated results from Mehl et al. model [19].

Using a minimum detectable absorbance of $0.1 \%$, a path-length of $14 \mathrm{~cm}$ and the measured absorption cross-sections, the detection limit of this sensor, at $1300 \mathrm{~K}$ and $1 \mathrm{~atm}$, will be $\sim 75 \mathrm{ppm}$ for $\mathrm{CH}_{4}$ and $\sim 101$ ppm for $\mathrm{C}_{2} \mathrm{H}_{2}$. Detection limits for methane and acetylene as a function of temperature are summarized in Fig. S7 (Supplementary Material).

\section{Conclusions}

A new laser sensor, based on scanned-wavelength direct absorption spectroscopy, was developed by employing a novel IH-QCL (integrated heater - quantum cascade laser). The IH-QCL allows faster control of the AR (active region) temperature and a wider tuning capability compared to conventional DFB lasers. The IH-QCL exhibits some very interesting features that could be quite useful for various absorption 
305 spectroscopy applications. When the IH and AR currents are modulated in phase, the laser provides wider 306 wavelength tuning than would be possible with the typical AR current modulation. When the two 307 modulations are out of phase, the wavelength tuning is suppressed and this may be of use for experiments 308 which seek to maximize intensity modulation with minimal wavelength tuning. On the other hand, the 309 laser intensity modulation amplitude is reduced significantly with IH modulation alone, which could be 310 taken advantage of in wavelength modulation spectroscopy to minimize residual amplitude modulation.

311 To demonstrate the potential of this laser architecture for combustion applications, the laser was used to 312 make simultaneous measurements of methane and acetylene in a shock tube with a time resolution of 20 $313-40 \mu \mathrm{s}$. Measurements carried out during the high-temperature pyrolysis of iso-octane showed the high 314 potential of multi-species detection in shock tube chemical kinetics experiments. Future work will be 315 aimed at exploiting the capabilities of this laser for high-pressure measurements and for sensing species 316 with broadband spectral features.

318 Acknowledgment

319 Research reported in this publication was funded by the Office of Sponsored Research at King Abdullah 320 University of Science and Technology (KAUST).

\section{References}

324 [1] J.B. McManus, M.S. Zahniser, D.D. Nelson, J.H. Shorter, S. Herndon, E. Wood, R. Wehr, Optical Engineering, $32549(11)(2010) 111124$.

326 [2] L. Tao, K. Sun, M.A. Khan, D.J. Miller, M.A. Zondlo, Optics express, 20 (27) (2012) 28106-28118. 
327 [3] M. Wei, R. Kan, B. Chen, Z. Xu, C. Yang, X. Chen, H. Xia, M. Hu, Y. He, J. Liu, Applied Physics B, 123 (5) 328 (2017) 149.

329 [4] T. Beyer, M. Braun, A. Lambrecht, Journal of applied physics, 93 (6) (2003) 3158-3160.

330 [5] G. Wysocki, A. Kosterev, F. Tittel, Applied Physics B, 80 (4-5) (2005) 617-625.

331 [6] M. Silva, D. Sonnenfroh, D. Rosen, M. Allen, A. O’keefe, Applied Physics B, 81 (5) (2005) 705-710.

332 [7] I. Patel, V.P. Rajamanickam, A. Bertoncini, F. Pagliari, L. Tirinato, S.P. Laptenok, C. Liberale, in: Optical 333 Trapping Applications, Optical Society of America, 2017, pp. JTu4A. 21.

334 [8] X. Chao, J. Jeffries, R. Hanson, Applied Physics B, 106 (4) (2012) 987-997.

335 [9] R.S. Chrystie, E.F. Nasir, A. Farooq, Proceedings of the Combustion Institute, 35 (3) (2015) 3757-3764.

336 [10] R. Kazarinov, Sov. Phys.-Semicond., 5 (4) (1971) 707-709.

337 [11] A. Bismuto, Y. Bidaux, C. Tardy, R. Terazzi, T. Gresch, J. Wolf, S. Blaser, A. Muller, J. Faist, Optics express, $33823(23)(2015) 29715-29722$.

339 [12] DRS Daylight Solutions, https://www.daylightsolutions.com/product/cw-mhf/.

340 [13] NKT Photonics, http://www.nktphotonics.com/lasers-fibers/en/product/superk-mir-mid-ir-supercontinuum$341 \underline{\text { laser/. }}$

342 [14] J. Li, W. Chen, H. Fischer, Applied Spectroscopy Reviews, 48 (7) (2013) 523-559.

343 [15] S. Welzel, F. Hempel, M. Hübner, N. Lang, P.B. Davies, J. Röpcke, Sensors, 10 (7) (2010) 6861-6900.

344 [16] K. Utsav, E.F. Nasir, A. Farooq, Applied Physics B, 120 (2) (2015) 223-232.

345 [17] M. Sajid, T. Javed, A. Farooq, Journal of Quantitative Spectroscopy and Radiative Transfer, 155 (2015) 6634674.

347 [18] M.B. Sajid, T. Javed, A. Farooq, Combustion and Flame, 164 (2016) 1-9.

348 [19] M. Mehl, H. Curran, W. Pitz, C. Westbrook, in, Lawrence Livermore National Laboratory (LLNL), Livermore, 349 CA, 2009.

350 [20] R. Design, CHEMKIN-PRO 15131; Reaction Design: San Diego, CA, 2016.

351 [21] I.E. Gordon, L.S. Rothman, C. Hill, R.V. Kochanov, Y. Tan, P.F. Bernath, M. Birk, V. Boudon, A. Campargue, 352

K. Chance, Journal of Quantitative Spectroscopy and Radiative Transfer, 203 (2017) 3-69. 
353 [22] S.W. Sharpe, T.J. Johnson, R.L. Sams, P.M. Chu, G.C. Rhoderick, P.A. Johnson, Applied spectroscopy, 58 354 (12) (2004) 1452-1461.

355 [23] J. Badra, A.E. Elwardany, F. Khaled, S.S. Vasu, A. Farooq, Combustion and Flame, 161 (3) (2014) 725-734.

356 [24] J. Badra, A.E. Elwardany, A. Farooq, Physical Chemistry Chemical Physics, 16 (24) (2014) 12183-12193.

357 [25] S.M. Sarathy, T. Javed, F. Karsenty, A. Heufer, W. Wang, S. Park, A. Elwardany, A. Farooq, C.K. Westbrook, 358 W.J. Pitz, Combustion and Flame, 161 (6) (2014) 1444-1459.

359 [26] K. Gurel, S. Schilt, A. Bismuto, Y. Bidaux, C. Tardy, S. Blaser, T. Gresch, T. Sudmeyer, in: CLEO: 360 Applications and Technology, Optical Society of America, 2016, pp. ATh1J. 2.

361 [27] E.L. Petersen, R.K. Hanson, Shock Waves, 15 (5) (2006) 333-340.

362

List of figure captions:

\begin{tabular}{|c|c|}
\hline Figure No. & Caption \\
\hline Fig. 1 & Simulated spectra for a $2.5 \% \mathrm{CH} 4,1.5 \% \mathrm{C} 2 \mathrm{H} 2$, balance air mixture. \\
\hline Fig. 2 & Optical schematic of the experimental setup. \\
\hline Fig. 3 & $\begin{array}{l}\text { Wavelength tuning range of the IH-QCL at different modulation frequencies in three } \\
\text { different tuning manners. }\end{array}$ \\
\hline Fig. 4 & $\begin{array}{l}\text { Wavelength tuning range as a function of phase difference between AR and IH tuning } \\
\text { waves. }\end{array}$ \\
\hline Fig. 5 & Modulation amplitude with AR tuning and IH tuning separately. \\
\hline
\end{tabular}


Fig. 6

Fig. 7

Fig. 8
Measured absorption cross-sections of methane and acetylene at the two selected peaks by using 3\% $\mathrm{CH}_{4} / \mathrm{Ar}$ and $5 \% \mathrm{C}_{2} \mathrm{H}_{2} /$ Ar mixtures.

Measured mole-fraction time-histories for 3.1\% $\mathrm{CH}_{4} / 4.9 \% \mathrm{C}_{2} \mathrm{H}_{2} /$ Ar mixture.

Mole fraction results of methane and acetylene for two reflected-shock experiments at 1616 $\mathrm{K}, 1.20$ bar and $1965 \mathrm{~K}, 1.01$ bar. $\underline{\text { List of supplementary material: }}$

\begin{tabular}{ll}
\hline $\begin{array}{l}\text { Supplementary } \\
\text { materials }\end{array}$ & Caption \\
\hline
\end{tabular}

Fig. S1

Chemkin simulation results of the pyrolysis of $2 \%$ iso-octane/Ar mixture at an initial temperature of $1800 \mathrm{~K}$ and pressure of $1 \mathrm{~atm}$.

Fig. S2

Fig. S3

Fig. S4

Fig. S5

Fig. S6

Fig. S7
Absorbance of the species shown in Fig. S1. These are calculated using the PNNL database with a mole fraction of $1 \mathrm{ppm}$ and a path-length of $1 \mathrm{~m}$ at a temperature of $25 \mathrm{C}$ and a pressure of $1 \mathrm{~atm}$.

Experimental absorbance profile for a mixture of $1.4 \% \mathrm{CH}_{4}, 0.77 \% \mathrm{C}_{2} \mathrm{H}_{2}$ and balance $\mathrm{N}_{2}$ at room temperature (294K), pressure of 102 torr and effective path-length of $14 \mathrm{~cm}$.

Representative laser signal for cross-section measurement of methane.

A typical one-cycle laser transmitted signal (left) and its absorption profile (right) for the reflected shock of $1388 \mathrm{~K}$ and 1.07 atm.

Measured absorption profiles of 38 cycles for (a) the reflected shock of $1616 \mathrm{~K}$ and $1.2 \mathrm{~atm}$ and (b) the reflected shock of $1965 \mathrm{~K}$ and $1.0 \mathrm{~atm}$.

Detection limits in methane and acetylene at different temperatures from $1300 \mathrm{~K}$ to $1700 \mathrm{~K}$ and a pressure of $1 \mathrm{~atm}$. 